\title{
Alcoholic cirrhosis is a good indication for liver transplantation, even for cases of recidivism
}

\author{
G-P Pageaux, J Michel, V Coste, P Perney, P Possoz, P-F Perrigault, F Navarro,
} J-M Fabre, J Domergue, P Blanc, D Larrey

\begin{abstract}
BackgroundlAims-Alcoholic cirrhosis remains a controversial indication for liver transplantation, mainly because of ethical considerations related to the shortage of donor livers. The aim of this study was to review experience to date, focusing on survival rates and complications, and the effect of alcohol relapse on outcome and alterations in marital and socioprofessional status.

Methods-The results for 53 patients transplanted for alcoholic cirrhosis between 1989 and 1994 were compared with those for $\mathbf{4 8}$ patients transplanted for nonalcoholic liver disease. The following variables were analysed: survival, rejection, infection, cancer, retransplantation, employment and marital status, alcoholic recurrence. The same variables were compared between alcohol relapsers and non-relapsers.

Results-Recovery of employment was the only significantly different variable between alcoholic (30\%) and non-alcoholic patients $(60 \%)$. Two factors influenced survival in the absence of alcohol recidivism: age and abstinence before transplantation. For all other variables, there were no differences between alcoholic and non-alcoholic patients, and, within the alcoholic group, between relapsers and non-relapsers. The recidivism rate was $32 \%$.

Conclusion-The data indicate that liver transplantation is justified for alcoholic cirrhosis, even in cases of recidivism, which did no affect survival and compliance with the immunosuppressive regimen. These good results should help in educating the general population about alcoholic disease.

(Gut 1999;45:421-426)
\end{abstract}

Keywords: liver; transplantation; alcohol; cirrhosis

Alcoholic cirrhosis is the leading cause of end stage liver disease in western countries, and many alcoholic patients may potentially benefit from liver transplantation if they fulfil the usual criteria for this procedure. ${ }^{1}$ Many centres have reported that alcoholic patients who undergo liver transplantation progress as well as patients transplanted for non-alcoholic liver diseases. ${ }^{2-11}$ However, we have to take into account the other diseases, especially neurological and cardiovascular, caused by alcoholism, the improvements that occur after alcohol with- drawal rendering transplantation unnecessary, and the risk of alcoholic relapse after transplantation which could potentially be responsible for poor compliance with the immunosuppressive regimen and result in graft failure. ${ }^{12}$ Moreover, this fear of a high rate of alcohol recidivism focuses on ethical considerations related to the shortage of donor livers, and this problem is that of inequality: some patients will receive a graft while others will not. Heavy drinking leading to alcoholic cirrhosis is widely regarded as morally wrong and there has been some discrimination against alcoholics in liver transplant programmes and in the general population sensitive to organ donation. ${ }^{13}$ All these considerations were discussed during the consensus conference on indications for liver transplantation held in Paris in 1993. It was stated that the indications for liver transplantation in alcoholic cirrhotic patients were limited to "patients whose liver disease remains serious despite alcohol withdrawal, without any consensus on the ideal period of abstinence: 3 to 6 months or more". ${ }^{14}$

The present retrospective study reviews our experience in liver transplantation for alcoholic cirrhosis, focusing on survival rates and complications compared with patients who underwent transplantation for non-alcoholic liver disease, and the effect of alcohol relapse on outcome and alterations in marital and socioprofessional status after transplantation.

\section{Materials and methods}

PATIENT SELECTION

Between March 1989 and December 1994, 53 patients underwent liver transplantation for alcoholic cirrhosis at the liver transplant unit of the University of Montpellier. Two of the recipients suffered from acute alcoholic hepatitis complicating the cirrhosis. Six patients presented with hepatocellular carcinoma and alcoholic cirrhosis. Patients were considered for transplantation if liver function suggested a poor prognosis, correlated to Child-Pugh's score B or C and/or cases of spontaneous bacterial peritonitis, refractory ascites, and chronic encephalopathy. All patients underwent an extensive and multidisciplinary evaluation, performed by a hepatologist, an anaesthetist and a surgeon, and included a careful cardiac and respiratory work up. No formal psychiatric evaluation was performed during this period because we had not identified a specialised psychiatrist interested in these patients. Before transplantation was considered, abstinence from alcohol consumption was required to be sure that there was no improvement in liver 
Table 1 Diagnoses of comparison group (48 patients)

\begin{tabular}{lc}
\hline Indication for liver transplant & Number \\
\hline HCV cirrhosis & 18 \\
HBV or BD cirrhosis & 10 \\
Primary biliary cirrhosis & 7 \\
Primary sclerosing cholangitis & 4 \\
Autoimmune cirrhosis & 1 \\
Wilson's disease & 1 \\
Crytptogenetic cirrhosis & 7 \\
\hline
\end{tabular}

$\mathrm{HCV}$, hepatitis C virus; HBV, hepatitis B virus; BD, B-delta.

function without alcohol intake. Furthermore, most importantly, patient acceptance of his/her alcoholism was expected. We paid particular attention to the motivation of the candidate and the family. Using all these criteria, 19 alcoholic patients referred to our programme were refused transplants, 11 on medical grounds and eight because of problems of alcoholism.

The comparison group consisted of 48 contemporaneous recipients of a primary graft transplanted during the same period by the same surgeons for non-alcoholic end stage liver disease (table 1), except for fulminant hepatitis and cancers without liver disease. Eight patients presented with hepatocellular carcinoma and non-alcoholic liver disease. Five patients referred to our programme were refused transplants on medical grounds.

\section{PATIENT FOLLOW UP}

All patients received blood group compatible grafts and were treated with the same immunosuppressive regimen, which combined cyclosporin, steroids, and azathioprine. Blood levels of cyclosporin were maintained between 100 and $300 \mathrm{mg} / \mathrm{ml}$ by the use of a monoclonal radioimmunoassay. In cases of renal dysfunction either during or after transplantation, anti-thymocyte globulins replaced cyclosporin for 8-10 days. A diagnosis of acute cellular and chronic rejection was based on the clinical and biochemical course in combination with histological evaluation of a percutaneous liver biopsy specimen. Episodes of rejection that were unresponsive to bolus corticosteroids were treated with preparations of anti-T cell monoclonal antibodies (OKT3) for 10-14 days. Chronic rejection was treated with tacrolimus if available or retransplantation.

Clinical signs and cultures were necessary for diagnosis of bacterial and fungal infections. Cytomegalovirus infection was detected by use of viral culture and antigenaemia assay; isolation of cytomegalovirus from blood and/or cytomegalovirus antigenaemia presence greater than five positive cells provided definitive evidence of cytomegalovirus infection requiring gancyclovir therapy. ${ }^{15}$ Clinical examination and culture allowed diagnosis of herpes simplex virus infection. Epstein-Barr virus infection was defined by Epstein-Barr virus serology variations. Epstein-Barr virus presence in lymphoproliferative tissues was proved by in situ hybridisation for Epstein-Barr virus early RNA.

Patients had routine outpatient clinical and biochemical examinations by a member of the transplant team. The follow up intervals were usually once a week during the first month after leaving hospital, twice a month during the second and third months, monthly during the first year, every two or three months thereafter, and anytime a problem occurred. At each visit, patients were questioned on alcohol consumption (only those transplanted for alcoholic cirrhosis), compliance with immunosuppressive drugs, employment (employed, unable to work), and marital status (married or living with a partner, divorced, single). These data were sought and recorded by a medical member of the transplant team (GPP), using an informal interview with the patient and the family. Recidivism was defined as any alcohol use after liver transplantation. For the purpose of the study, alcohol consumption levels were defined by the following criteria: mild drinking, less than $20 \mathrm{~g}$ a day; moderate, 20-40 g a day; excessive, more than $40 \mathrm{~g}$ a day. Checks for serum or urine alcohol were not systematically performed. If there was any doubt about recidivism not admitted by the patient, a liver biopsy was performed. All data were reviewed from medical files for each patient.

\section{STATISTICAL ANALYSIS}

Comparisons of quantitative variables were performed using Student's $t$ test or the MannWhitney $U$ test. Comparisons of qualitative variables were performed using the MantelHaenszel $\chi^{2}$ test or Fisher's exact test. Alcohol recidivism rate and employment and marital status were considered to be non-fatal clinical end points. Only patients surviving more than three months (47 of the 53 patients) were considered for analysis of non-fatal clinical end points. All patients were included in the assessment of patient survival, rejection rate, and infections. To take into account the length of time before an event (death or recidivism), we used the Kaplan-Meier method. The survival curves were compared by use of the log rank non-parametric test. Multivariate analysis was performed with the Cox model. $\mathrm{p}<0.05$ was considered as significant. All results up to 31 December 1997 were analysed.

\section{Results}

PATIENT CHARACTERISTICS BEFORE

TRANSPLANTATION

Of the 53 patients transplanted for end stage alcoholic liver disease, 44 were men and nine were women, with an average age at the time of transplantation of 48.8 years (range 31 to 63). Forty three $(81 \%)$ were Child-Pugh's score C, and $10(19 \%)$ were score B. Fifty one $(96 \%)$ were abstinent before transplantation, 15 for less than six months and 36 for more than six months. Of the 48 patients transplanted for non-alcoholic liver disease, 23 were men and 25 were women, with an average age at the time of transplantation of 48.8 years (range 18 to 66). Thirty (63\%) were Child-Pugh's score C, $13(27 \%)$ were score B, and five $(10 \%)$ were score A. There was a significant difference between the two groups with regard to sex repartition $(p=0.0002)$. There was no significant difference between the two groups with regard to hospitalisation status at the time of 


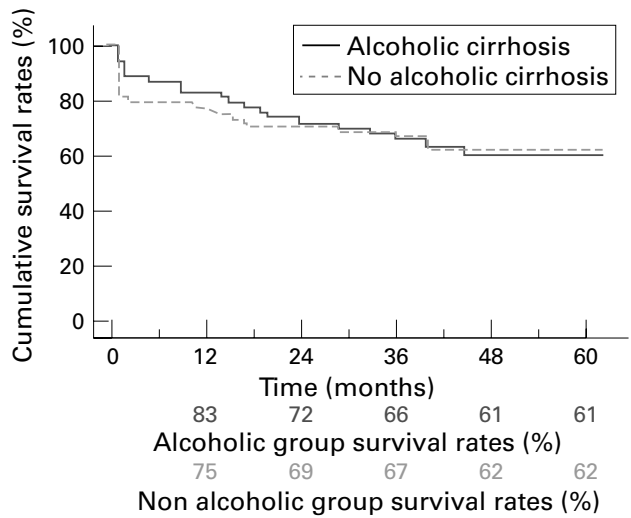

Figure 1 Overall survival rates for patients with alcoholic cirrhosis $(n=53)$ and those without alcoholic cirrhosis ( $n$ $=48$ ) undergoing liver transplantation. There was no difference between the two groups $(p=0.97)$.

the transplant, history of upper gastrointestinal tract haemorrhage, and history of spontaneous bacterial peritonitis.

SURVIVAL ANALYSIS

The mean follow up for the 53 alcoholic patients was 42.1 months (range 1 to 100 ), but if we consider the 47 patients who lived for more than three months, it was 47.3 months (range 5 to 100). There were 33 long term survivors with a mean follow up of 57.8 months (range 36 to 100 ). The overall survival rate at one, two, three, and five years was $75,69,67$, and $62 \%$ respectively (fig 1 ).

In the 48 non-alcoholic patients, the mean follow up was 46.6 months (range 1 to 93 ), but if we consider the 38 patients who lived for more than three months, it was 58.6 months (range 10 to 93 ). The overall survival rate at one, two, three, and five years was $83,72,66$, and $61 \%$ respectively (fig 1 ). There was no significant difference between the two groups.

Table 2 reports the causes of death in the two groups. Of the six alcoholic patients who died from cancer, three had post-transplant lymphoproliferative disorders, one had pancreatic cancer, one had breast cancer, and one had larynx cancer.

REJECTION

In the alcoholic group, the acute rejection rate was $47.1 \%$ and the chronic rejection rate was $5.6 \%$. Only one patient in this group experienced one acute rejection episode related to poor compliance with the immunosuppressive regimen; this was successfully treated with a

Table 2 Causes of death and survival period

\begin{tabular}{lll}
\hline Causes of death & Alcoholic group $(n=20)$ & Non-alcoholic group $(n=18)$ \\
\hline $\begin{array}{l}\text { Hospital mortality (death }<3 \\
\text { months) }\end{array}$ & 6 & 10 \\
$\begin{array}{l}\text { Hepatocellular carcinoma } \\
\quad \text { recurrence }\end{array}$ & $3(9,10,20)$ & $3(14,14,36)$ \\
$\begin{array}{l}\text { Chronic rejection } \\
\text { Cardiopulmonary disease }\end{array}$ & $2(13,14)$ & $2(16,32)$ \\
Cancer & $1(31)$ & $1(7)$ \\
Trauma & $6(5,14,21,32,38,44)$ & - \\
HCV recurrence & $2(16,36)$ & - \\
Mesenteric infarction & - & $1(13)$ \\
& - & $1(46)$
\end{tabular}

Values in parentheses are survival period in months.

$\mathrm{HCV}$, hepatitis $\mathrm{C}$ virus.
Table 3 Infections after liver transplantation

\begin{tabular}{lll}
\hline Type of infection & $\begin{array}{l}\text { Alcoholic group } \\
(\%)(n=53)\end{array}$ & $\begin{array}{l}\text { Non-alcoholic group } \\
(\%)(n=48)\end{array}$ \\
\hline Bacterial & 35.8 & 29.1 \\
Fungal & 9 & 6 \\
Viral & & \\
CMV & 45 & 35 \\
HSV & 9.4 & 0.4 \\
EBV & 9.4 & 8.3
\end{tabular}

$\mathrm{CMV}$, cytomegalovirus; HSV, herpes simplex virus; EBV, Epstein-Barr virus.

Table 4 Reasons for retransplantation

\begin{tabular}{lll}
\hline Reason & $\begin{array}{l}\text { Alcoholic group } \\
(n=6)\end{array}$ & $\begin{array}{l}\text { Non-alcoholic } \\
\text { group }(n=7)\end{array}$ \\
\hline Chronic rejection & 3 & 2 \\
Hepatic artery thrombosis & 2 & 2 \\
Biliary complications & 1 & - \\
Non-functioning graft & - & 2 \\
HCV recurrence & - & 1 \\
\hline
\end{tabular}

$\mathrm{HCV}$, hepatitis $\mathrm{C}$ virus.

corticosteroid bolus. This patient had alcoholism recurrence and was irregularly taking drugs. No liver biopsy follow up was performed on this patient.

In the non-alcoholic group, the acute rejection rate was $43.7 \%$ and the chronic rejection rate was $6.2 \%$. There was no significant difference between the two groups, in either frequency of rejection or severity and treatment efficacy.

INFECTION

Table 3 gives results on viral, bacterial, and fungal infections in the two groups. There was no significant difference between the two groups. With respect to fungal infections, we observed the same significant difference in the two groups between living and dead patients: for the 53 alcoholic patients, one $(3 \%)$ of the 33 living patients and four $(20 \%)$ of the 20 dead patients had fungal infections $(\mathrm{p}=0.04)$; for the 48 non-alcoholic patients, none of the 30 living patients and three $(16 \%)$ of the 18 dead patients had fungal infections $(\mathrm{p}=0.04)$.

CANCERS

In the alcoholic group, nine patients (17\%) experienced cancer de novo: three posttransplant lymphoproliferative disorder, two larynx cancer, one Kaposi syndrome, one skin cancer, one breast cancer, one pancreatic cancer. In the non-alcoholic group, three patients (6\%) experienced cancer de novo: one posttransplant lymphoproliferative disorder, one larynx cancer, one skin cancer. The three patients who developed larynx cancer were smokers. However, smoking was not systematically analysed in the two groups.

RETRANSPLANTATION

In the alcoholic group, six patients $(11 \%)$ were retransplanted, compared with seven $(14 \%)$ in the non-alcoholic group. This was not a significant difference. Table 4 reports the causes of retransplantation in the two groups.

EMPLOYMENT AND MARITAL STATUS

For these non-fatal clinical end points, we only considered patients living for more than three months. After transplantation, $26 \%$ of the 
alcoholic patients compared with $45 \%$ of the non-alcoholic patients regained employment $(p=0.04)$. If we take into account only the long term living patients, $30 \%$ of the alcoholic patients compared with $60 \%$ of the nonalcoholic patients regained employment $(\mathrm{p}=$ 0.02).

With respect to marital status, $16 \%$ of the alcoholic patients compared with $8 \%$ of the non-alcoholic patients were either divorced or separated from their partner $(p=0.19 ; \mathrm{NS})$. If we take into account only the long term living patients, $18 \%$ of the alcoholic patients compared with $7 \%$ of the non-alcoholic patients were in this situation $(\mathrm{p}=0.14 ; \mathrm{NS})$.

\section{ALCOHOLIC RECURRENCE}

We did not systematically analyse drinking behaviour in the non-alcoholic patients. Analysis was performed on the alcoholic patients who survived for more than three months. Although strict instructions on total abstinence after transplantation were given to alcoholic patients before the operation, 15 of the 47 recipients $(32 \%)$ had resumed alcohol consumption during a median follow up of 47.3 months. Thirteen of these 15 patients $(86 \%)$ were considered to be abstinent before transplantation. The two non-abstinent patients had acute alcoholic hepatitis. The estimated risk for alcoholism recurrence after one, two, and three years was 27,32 , and $32 \%$ respectively.

Seven of the 15 drinking patients $(46.6 \%)$ compared with two of the 32 non-drinking patients $(6 \%)$ had been abstinent for less than six months before transplantation $(\mathrm{p}=0.04)$. Of the 15 patients who relapsed, two returned to mild, eight to moderate, and five to excessive drinking. Of the two patients with acute alcoholic hepatitis before transplantation, one returned to excessive drinking and it was the cause of death; the other returned to moderate and intermittent drinking.

We reassessed all the fatal and non-fatal clinical end points of the 47 alcoholic liver recipients to compare those who relapsed and those who did not (table 5).

All biopsy specimens taken from 23 of the 33 long term survivors of the alcoholic group were reviewed. Data in table 6 represent the histological features of alcohol abstinents and users. None of the alcohol users had a normal biopsy result. Steatosis was the most common abnormality in the two groups. One alcoholic patient developed acute alcoholic hepatitis and

Table 5 Effect of recurrent alcoholism on clinical end points

\begin{tabular}{llll}
\hline Clinical end point & $\begin{array}{l}\text { Alcohol relapse } \\
(n=15)\end{array}$ & $p$ Value & $\begin{array}{l}\text { No alcohol relapse } \\
(n=32)\end{array}$ \\
\hline Age (years) & $44(31-54)$ & 0.004 & $50(33-62)$ \\
Female/male & $4 / 11$ & 0.4 & $5 / 27$ \\
Overall survival & $12(80)$ & 0.09 & $18(55)$ \\
Mean survival (months) & $54(17-94)$ & 0.08 & $44(5-100)$ \\
Retransplantation & 0 & 0.16 & $5(15)$ \\
Acute rejection & $5(33)$ & 0.52 & $16(50)$ \\
Chronic rejection & 0 & 0.22 & $3(9)$ \\
Cytomegalovirus & $6(40)$ & 0.22 & $16(50)$ \\
Bacterial infections & $4(26)$ & 0.46 & $12(37)$ \\
Fungal infections & $1(6)$ & 1.00 & $2(6)$ \\
Employment & $4(26)$ & 0.96 & $9(28)$ \\
Divorce or separation & $5(33)$ & 0.04 & $3(9)$ \\
\hline
\end{tabular}

Values in parentheses are percentages except for age and mean survival where they are ranges.
Table 6 Histological features in liver biopsy specimens taken after transplantation from long term survivors in the alcoholic group

\begin{tabular}{lll}
\hline Biopsy result & $\begin{array}{l}\text { Abstainers } \\
(n=14)\end{array}$ & $\begin{array}{l}\text { Users } \\
(n=9)\end{array}$ \\
\hline Normal & 3 & 0 \\
Steatosis & 5 & 8 \\
Acute alcoholic hepatitis & 0 & 1 \\
Lobular hepatitis & 3 & 2 \\
Chronic active hepatitis & 0 & 1 \\
Portal inflammation & 2 & 0 \\
Cholangitis & 2 & 0 \\
\hline
\end{tabular}

refused any help to achieve sobriety. Lobular hepatitis related to hepatitis $\mathrm{C}$ virus reinfection of the graft was seen in five patients. One patient developed chronic active hepatitis related to acquired hepatitis $\mathrm{B}$ virus infection. No patient showed any signs of acute or chronic rejection.

Two variables were statistically significant. Patients who relapsed were younger (44 years) than those who did not (50 years) $(p=0.004)$, and there were more divorces or separations for relapsing patients $(33 \%)$ than non-relapsing patients $(9 \%)(\mathrm{p}=0.04)$.

We used multivariate analysis to look for factors that influenced overall survival and survival without alcohol recidivism. Three significant variables were assessed by univariate analysis for overall survival: age, fungal infection, and chronic rejection. None was found to be an independent prognostic factor (age, $\mathrm{p}=$ 0.10 ; fungal infection, $\mathrm{p}=0.10$; chronic rejection, $\mathrm{p}=0.09$ ). The three significant variables for survival without alcohol recidivism, assessed by univariate analysis, were: age, abstinence before transplantation, and marital status. Marital status was not an independent prognostic factor $(p=0.15)$. Age was significant $(p=0.01)$ : risk to relapse was $\times 0.9$ for each increase of one year. Abstinence before transplantation was significant: risk to relapse $\times$ 5.5 for abstinence less than six months $(p=$ $0.005)$ and $\times 31$ without abstinence $(\mathrm{p}=$ $0.003)$.

\section{Discussion}

The major argument against widespread use of liver transplantation for patients with alcoholic cirrhosis has been the fear of a high rate of recidivism leading to loss of the graft. We have tried to emphasise, on the one hand, the lack of medical arguments for removing alcoholics from liver transplant programmes and, on the other hand, the moral discrimination against alcoholics.

Except for one initial report of poor survival after liver transplantation for alcoholic liver disease, ${ }^{16}$ all recent studies have clearly shown that mortality after transplantation for alcoholic patients does not differ from that for non-alcoholic recipients. ${ }^{2-11} 1718$ Our data are in agreement with these previous studies (table 7 ). Moreover, the overall one and five year survival rates (75 and $62 \%$ respectively) do not differ from those for patients transplanted for non-alcoholic liver disease (83 and $61 \%$ respectively). In our experience, causes of death were similar in the two groups, except for trauma and cancer which were only reported 
Table 7 Results in the literature on liver transplantation for alcoholic cirrhosis

\begin{tabular}{|c|c|c|c|c|c|c|c|c|c|c|c|}
\hline \multirow[b]{2}{*}{ Reference } & \multirow[b]{2}{*}{ Period } & \multirow[b]{2}{*}{ Number } & \multirow{2}{*}{$\begin{array}{l}\text { Follow up } \\
\text { (months) }\end{array}$} & \multicolumn{4}{|c|}{ Survival (\%) } & \multirow{2}{*}{$\begin{array}{l}\text { Long term } \\
\text { survivors }\end{array}$} & \multirow{2}{*}{$\begin{array}{l}\text { Recidivism } \\
(\%)\end{array}$} & \multirow{2}{*}{$\begin{array}{l}\text { Graft } \\
\text { dysfunction } \\
\text { related to } \\
\text { recidivism (\%) }\end{array}$} & \multirow{2}{*}{$\begin{array}{l}\text { Deaths related } \\
\text { to recidivism } \\
\text { (\%) }\end{array}$} \\
\hline & & & & 1 year & 2 year & 3 year & 5 year & & & & \\
\hline Bird and coworkers ${ }^{3}$ & $1980-1989$ & 24 & $\mathrm{ND}(4-84)$ & 66 & & & & 18 & 22 & ND & 0 \\
\hline Kumar and coworkers ${ }^{2}$ & $1982-1988$ & 73 & ND & 74 & & 62 & & 52 & 11.5 & 2 & 2 \\
\hline Knechtle and coworkers ${ }^{4}$ & $1984-1990$ & 41 & ND & 83 & & & 71 & 30 & 13 & 0 & 0 \\
\hline Berlakovitch and coworkers ${ }^{7}$ & $1982-1993$ & 58 & 33 & 71 & & & 63 & 44 & 31 & 16 & 4.5 \\
\hline Osorio and coworkers ${ }^{8}$ & $1988-1991$ & 43 & 21 & 100 & & & & 37 & 19 & ND & 0 \\
\hline Raakow and coworkers ${ }^{9}$ & 1988-1994 & 78 & 25 & 96 & & & 85 & ND & 22 & ND & 2.5 \\
\hline Gerhardt and coworkers ${ }^{10}$ & 1985-1991 & 67 & 47 & \multicolumn{3}{|c|}{67 overall } & & 41 & 49 & & 4.4 \\
\hline Foster and coworkers ${ }^{18}$ & 1986-1994 & 88 & 49 & 79 & 75 & & & 63 & 22 & 17 & 5 \\
\hline Lucey and coworkers ${ }^{11}$ & 1987-1991 & 59 & 63 & 80 & & & 77 & 50 & 34 & & \\
\hline Anand and coworkers ${ }^{17}$ & $1987-1994$ & 39 & & 79 & & & 79 & & 13 & & \\
\hline Gish and coworkers ${ }^{6}$ & 1988-1991 & 29 & 24 & 93 & & & & 29 & 21 & ND & 0 \\
\hline Doffoel and coworkers ${ }^{5}$ & $1985-1991$ & 75 & 29 & 80 & & 68 & & 57 & 26 & & \\
\hline Pageaux and coworkers & 1989-1994 & 53 & 42 & 75 & 69 & 67 & 62 & 47 & 32 & 4 & 2 \\
\hline
\end{tabular}

$\mathrm{ND}$, not determined.

for alcoholics. One of the two traumas was directly caused by excessive alcohol intake and the other one was accidental. Of the cancers, two involved the larynx. It is well known that alcoholic patients, who are often smokers, are highly prone to this kind of cancer, and the high prevalence of throat cancer after liver transplantation has already been reported. ${ }^{19}$ This fact has prompted careful otorhinolaryngology screening before transplantation. Even in recipients who have returned to alcohol abuse, the overall survival was as good as, if not better than (although not significantly), that in abstinent patients ( 80 and $55 \%$ respectively).

One main argument against transplantation for alcoholics is that the presumed high rate of recidivism would lead to poor adherence to the immunosuppressive regimen and premature loss of the graft. We assessed compliance with the immunosuppressive regimen by looking at the incidence of rejection. Again, there was no difference between alcoholics $(47.1 \%$ and $5.6 \%$ ) and non-alcoholics (43.7\% and 6.2\%) with respect to acute and chronic rejection respectively, just as we compared alcohol relapsers $(35 \%$ and $0 \%)$ and non-relapsers (50\% and $9 \%$ ). Moreover, we did not observe any cases of rejection in follow up liver biopsies after the transplant. In fact, little is known about actual compliance with the immunosupressive regimen in any patient who undergoes liver transplantation, and alcoholics do not differ from non-alcoholic patients.

Another point of interest is the occurrence of infections after liver transplantation. It is known that infections, especially bacterial and fungal, are linked to poor nutritional status. ${ }^{20}$ Alcoholic patients often suffer from poor nutrition compared with non-alcoholic patients. In our study, we observed no difference between the two groups of patients with regard to infections. However, fungal infections, whatever the patient, had a very bad prognosis, occurring in patients with multiple complications during the first three months of follow up.

The data on employment status are discouraging. Considering long term survivors, only $30 \%$ of alcoholics and $60 \%$ of non-alcoholics regained employment. However, among the alcoholics, there was no difference between relapsers and non-relapsers. We believe that these discouraging results are mainly related to the economic conditions in France and the high rate of unemployment. The significant difference between alcoholics and nonalcoholics seems to be related to the level of occupation before transplantation, which was lower in alcoholics than in non-alcoholics, and the poorer medical condition of alcoholics ( $81 \%$ Child-Pugh's score C) than nonalcoholics (63\% Child-Pugh's score C) before transplantation.

The rate of divorce or separation after transplantation was similar in the two groups but, significantly, it was higher in alcohol relapsers $(33 \%)$ than abstinents $(9 \%)$. In most of these cases of separation, liver transplantation itself was not the determining factor but it seemed to be the continuation of a disturbing affective situation worsened by alcoholism.

In our population, we observed a rather high recidivism rate of $32 \%$ for alcoholism after transplantation, and, if we consider the 33 long term survivors, the rate increases to $36 \%$. Several reasons can be evoked. Firstly, the long follow up of patients after transplantation can be cited: it is usually reported that the frequency of relapse increases as the duration of follow up is extended..$^{7811}$ However, in this study, we have found a large risk for recurrence at one and three years (27 and 32\% respectively). Another reason may be the strict follow up of transplant patients at our unit, which may have contributed to a more accurate detection of alcohol intake. We do not use telephone contact, and the recording of drinking episodes is carried out during consultations with members of the team, usually the same one for each patient in order to maintain personal relations. Unlike Howard et $a l^{21}$ who attributed their detection of a high rate of recidivism (19 of 20 long term alcoholic liver transplant survivors) to the use of an interviewer who was independent of the transplant group, we believe that members of the transplant team are in a better position to detect alcohol relapse. Whereas underestimation of self reported alcohol use is often suggested, we have to consider a possible overestimation because of the drastic definition of recidivism that we use, namely the report of any alcohol use after liver transplantation. The question is whether the most important outcome for the patient is to be alive five years after liver transplantation, rejection free, with 
low doses of toxic immunosuppressive drugs or being able to avoid, at any price, drinking $20 \mathrm{~g}$ of alcohol a day. We favour the first option. In fact, the consequences of alcohol recidivism after transplantation may not necessarily be deleterious to the patient him/herself, but may have a disastrous impact on the general population who may be influenced by moral arguments producing repercussions on organ donation, namely reservations about giving organs to patients who may drink again after transplantation. Therefore we need to explain to the public that alcoholism is not a vice but a disease.

However, at the same time, we have to try to identify during the selection procedure those patients at greatest risk of alcohol relapse. ${ }^{16}{ }^{17}$ We found that young age and short period of abstinence before transplantation (less than six months) influenced alcohol recidivism. Previous studies are contradictory. Most of them found that the length of abstinence before transplantation is the main predictor of behaviour afterwards. ${ }^{179}$ In contrast, Lucey et $a l^{11}$ found no significant differences at the time of evaluation between abstainers and alcohol users in age, sex, severity of liver dysfunction, median duration of abstinence, or local prognosis score. Foster et al ${ }^{18}$ found that psychosocial criteria, co-morbid substance use, and possibly family history have greater predictive value than the length of abstinence before transplantation. From our own experience and that reported by other teams, we believe that the most important factor in the preoperative period is the acceptance by the patient and his/ her family of his/her alcoholism. ${ }^{22}$ In addition, since 1995, a psychiatrist has been involved in the evaluation before and the follow up after transplantation, not only to assess psychological selection criteria, but, above all, to help the patients to understand and to face their alcoholic disease. We have to bear in mind the fear of alcoholic patients that "without the alcohol, they will literally fall apart and cease to function, if not cease to exist". ${ }^{23}$

Finally, analysis of biopsy specimens from the 33 long term survivors is interesting. There was no difference between abstinents and users, except for one patient with acute alcoholic hepatitis. We observed neither fibrosis nor cirrhosis. The length of the follow up cannot be the only argument put forward, as alcoholic cirrhosis recurrence 21 months after liver transplantation has been reported. ${ }^{24} \mathrm{Bad}-$ dour et $a l^{25}$ found four of 23 patients developed cirrhosis, detected by liver biopsy performed from 177 to 711 days after liver transplantation. No relation between fibrosis and immunosuppressive drugs has been found.

In conclusion, our data indicate that liver transplantation is justified for alcoholic cirrhosis. We observed an overall recidivism rate of
$32 \%$, which did not affect survival and compliance with the immunosuppressive regimen. Only $10.6 \%$ of the patients returned to excessive alcohol use (up to $40 \mathrm{~g}$ a day). Special attention must be given to young recipients who have the greatest risk of recidivism. The good results obtained for liver transplantation for alcoholic cirrhosis should help us to educate the general population about alcoholic disease.

1 Poynard T, Barthelemy P, Fratte S, et al. Evaluation of efficacy liver transplantation in alcoholic cirrhosis by a case-control study and simulated controls. Lancet 1994;344:502-7.

2 Kumar S, Stauber RE, Gavaler JS, et al. Orthotopic liver transplantation for alcoholic liver disease. Hepatology 1990; 11:159-64.

3 Bird GLA, O'Grady JG, Harvey FAH, et al. Liver transplantation in patients with alcoholic cirrhosis: selection criteria and rates of survival and relapse. BMF 1990;301:15-17.

4 Knechtle SJ, Fleming MF, Barry KL, et al. Liver transplantation for alcoholic liver disease. Surgery 1992;112:694703 .

5 Doffoel M, Fratte S, Vanlemmens C, et al. Results of liver transplantation in 75 French patients with alcoholic cirrhosis. Comparison with a non alcoholic group [abstract]. Hepatology 1992;16:A18.

6 Gish RG, Lee AH, Keffe EB, et al. Liver transplantation for patients with alcoholism and end-stage liver disease. $A m \mathcal{F}$ patients with alcoholism and end

7 Berlakovich GA, Steininger R, Herbst F, et al. Efficacy of liver transplantation for alcoholic cirrhosis with respect to recidivism and compliance. Transplantation 1994;58:560-5.

8 Osorio RW, Ascher NL, Avery M, et al. Predicting recidivism after orthotopic liver transplantation for alcoholic liver disease. Hepatology 1994;20:105-10.

9 Raakow R, Langrehr JM, Rohmann R, et al. Is orthotopic liver transplantation for end-stage alcoholic cirrhosis justified? Transplant Proc 1995;27:1241-2.

10 Gerhardt TC, Goldstein RM, Urschel HC, et al. Alcohol use following liver transplantation for alcoholic cirrhosis. Transplantation 1996;62:1060-3.

11 Lucey MR, Carr K, Beresford TP, et al. Alcohol use after liver transplantation in alcoholics: a clinical cohort follow-up study. Hepatology 1997;25:1223-7.

12 Sherlock S. Alcoholic liver disease. Lancet 1995;345:227-9.

13 Moss AH, Siegler J. Should alcoholics compete equally for liver transplantation? $\mathcal{F} A M A$ 1991;265:1295-8.

14 Consensus conference on indications of liver transplantation: June 22-23, 1993. Hepatology 1994; 20(suppl.):635-5.

15 The TH, Van den Berg AP, Van Son WY, et al. Monitoring for cytomegalovirus after organ transplantation: a clinical perspective. Transplant Proc 1993;25(suppl 4):5.

16 Scharsch Midt BF. Human liver transplantation: analysis of data on 540 patients from four centers. Hepatology 1984;4: 95-101.

17 Anand AC, Ferraz-Neto B, Nightingale P, et al. Liver transplantation for alcoholic liver disease: evaluation of a selection protocol. Hepatology 1997;25:1478-84.

18 Foster PF, Fabrega F, Karademir S, et al. Prediction of abstinence from ethanol in alcoholic recipients following abstinence from ethanol in alcoholic recipients fo
liver transplantation. Hepatology 1997;25:1469-77.

19 Vanlemmens C, Bresson-Hadni S, Monnet E, et al. De novo malignancy following orthotopic liver transplantation for alcoholic cirrhosis [abstract]. Hepatology 1995;22:A375.

20 Wade JJ, Rolando N, Hayllar V, et al. Bacterial and fungal infections after liver transplantation: an analysis of 284 patients. Hepatology 1995;21:1328-36.

21 Howard L, Fahy T, Wong P, et al. Psychiatric outcome in alcoholic liver transplant patients. F Med 1994;87:731-6.

22 Knechtle SJ, FlemingMF, Barry KL, et al. Liver transplantation in alcoholics: assessment of psychological health and tion in alcoholics: assessment of psychological hea

23 Georgi JM. Transplantation in patients with alcoholic liver disease. In: Killenberg PG, Clavien PA, eds. Medical care of the liver transplant patient. Oxford: Blackwell Science, 1997: 70-87.

24 Bernard PH, Le Bail B, Carles J, et al. Liver retransplantation for alcoholic cirrhosis recurring within a 21 -month period. Transpl Int 1996;9:524-5.

25 Baddour N, Demetris AJ, Shah G, et al. The prevalence rate of onset and spectrum of histologic liver disease in alcohol of onset and spectrum of histologic liver disease in alcohol
abusing liver allograft recipients [abstract]. Gastroenterology 1992;102:A779. 